\title{
Diagnóstico de la atención \\ a oportunidades de género. \\ Caso de la Universidad Iberoamericana
}

DiAGNOSIS OF ATTENTION TO GENDER OPPORTUNITIES.

A CASE OF UNIVERSIDAD IbEROAMERICANA

Prof. Dra. Sanie Amparo Romero de Velázquez ${ }^{1}$

Prof. Ms. Graciela Molinas Santana ${ }^{2}$

\section{RESUMEN}

El trabajo de indagación se embarcó en el proyecto de Diseño y Atención a las Oportunidades de Género en la Educación Superior, liderado por la Universidad de Alicante (España). El objetivo se centró en diagnosticar situación de género y las políticas de abordaje de igualdad de oportunidades que se identifican en la organización de la Universidad Iberoamericana. Para recabar información se recurrió, en primer lugar, al análisis documental de los datos estadísticos para obtener una descripción demográfica de la organización y, en segundo lugar, a entrevistas a directivos y docentes de la institución para obtener datos sobre la identificación de las políticas de igualdad de oportunidad de género en la institución. Como resultado se ha podido comprobar que la organización cuenta con mayoría de mujeres en todos los estamentos que la conforman; en cuanto a las políticas se comprobó que las mismas están invisibilizadas en abordajes particulares y requieren ser formalizadas

${ }^{1}$ Educadora. Abogada. Dra. en Derecho. Posgrado en Didáctica Universitaria. Investigadora de la UNIBE. Rectora de la Universidad Iberoamericana.

${ }^{2}$ Educadora. Licenciada en Ciencias de la Educación. Posgrado en Didáctica Universitaria. Magister en Ciencias de la Educación con énfasis en Gestión Institucional Investigadora de la UNIBE. 
en los documentos institucionales. El análisis y discusión de los resultados ha permitido concluir en que se requiere identificar como un problema la igualdad de oportunidades, dado que la condición de contar con la mayoría de mujeres en la organización puede estar incidiendo significativamente en esta situación por lo que convendría realizar una investigación sobre la representación que las mujeres de la universidad tienen sobre el abordaje de género y su relación con la atención a las oportunidades en la Educación Superior.

Palabras clave: género, igualdad de oportunidades, políticas, Educación Superior.

\begin{abstract}
This research project was conducted during the project of Design and Account to Gender Opportunities in Higher Education project, headed by Universidad de Alicante (Spain). It aimed at diagnosing the situation of gender and the equity of opportunities policies as stated in the organization of Universidad Iberoamericana. To gather information, a documentary analysis of statistical data for a demographic description of the organization was conducted first. Second, principals and teachers of the institution were interviewed to obtain data on identification of policies concerning equity of gender opportunities in the institution. As a result it was proved that the organization is mostly composed of women at all levels. Concerning policies, it was confirmed that they are invisible in particular approaches and they need to be formalized in institutional papers. The analysis and discussion of the results has allowed to conclude that it is necessary to identify the equity of opportunities as a problem, since having a majority of women in the organization may significantly affect this situation. It would be convenient to conduct a research about the representation women from the university have about gender policies and its relation to attending opportunities in higher education.
\end{abstract}

Keywords: gender, equity of opportunities, policies, higher education.

\title{
10 UNIBE $\mid \begin{aligned} & \text { REVISTA } \\ & \text { CIENTÍFICA }\end{aligned}$
}




\section{INTRODUCCIÓN}

La Universidad Iberoamérica, creada por la Ley $\mathrm{N}^{\circ} 1701$ del 2001, está ubicada en la ciudad de Asunción, capital de la República del Paraguay. El fundamento educativo sobre el cual de sustenta es el de fomentar el saber multi e interdisciplinar como medio y fin de la formación integral de los estudiantes, con el lema "con una mentalidad nueva para un mundo mejor". Actualmente, cuenta con una sede en la ciudad de San Lorenzo, ubicada en el departamento Central del país. La universidad tiene sus orígenes en el año 1972, con la apertura de un Taller Artístico Literario, para luego ir como institución educativa ofreciendo la formación Inicial, Escolar Básica y Educación Media. Fortalecida como institución educadora, avanzó en la instauración del Instituto de Formación Docente en el nivel terciario, que forma maestros y maestras ejecutores de la Reforma Educativa del Paraguay. Cabe resaltar el desarrollo de la institución en casi medio siglo, 46 años.

Entre los fines y objetivos generales de la universidad se puede identificar el tratamiento de género a partir de las siguientes expresiones:

Apoyar la capacitación del hombre y mujer profesionales para el mejor desenvolvimiento en sus quehaceres específicos y su proyección social como protagonistas del mejoramiento en la vida de la comunidad a la cual pertenecen.

Promover la extensión universitaria colaborando positivamente en la promoción del desarrollo integral del hombre y de la mujer.

Estos postulados enmarcan el abordaje de género que se van desarrollando en la institución a través de actividades como el convenio con la Liga Paraguaya de defensa de los Derechos de la Mujer, con la ONG Kuña Aty", organización que atiende y asesora a mujeres que se encuentran en situación de vulnerabilidad ante la violencia intrafamiliar; "Despacho de la Primera Dama de la Nación", "Secretaría Nacional de la Mujer", en emprendimientos de sensibilización a través de seminarios y disertaciones referente a la igualdad de género en los talleres de "Herramientas para progresar", destinados a los docentes, estudiantes y la comunidad en general. Además cuenta con el Programa de Responsabilidad Social "Prof. Doña Nidia Sanabria de Romero", cuyo objetivo (entre otros) se han otorgado becas a las mujeres seleccionadas por dichas organizaciones mencionadas para cursar las ofertas educativas ofrecidas por la institución. 
En este trabajo se ha utilizado como técnica de obtención de datos el análisis documental para determinar la descripción demográfica por género de la población que interactúa en la organización de la universidad como una las informaciones relevantes que permitirá inducir las maneras de relacionamientos que pudieran estar dándose entre mujeres y hombres en la institución. Además se ha recurrido a las entrevistas a actores claves de la universidad para contar con datos que permitirán identificar el abordaje de las políticas de igualdad de oportunidades de género que se
desarrollan en la institución.

El estudio realizado abarca toda la universidad, con las siguientes facultades: de Ciencias humanísticas y de la Educación, de Ciencias Contables y Administrativas, de Ciencias de la Comunicación, Tecnología e Informática, Ciencias Sociales y Jurídicas, Ciencias Exactas y de Ciencias de la Salud. Las categorías de análisis fueron definidas por la Universidad de Alicante, coordinadora del proyecto.

El análisis y discusión de los resultados ha permitido concluir que la población mayoritaria de la Universidad Iberoamérica está conformada por mujeres y que las políticas de oportunidad de igualdad de género requieren ser explicitadas en los documentos institucionales.

\section{MARCO TEÓRICO-CONCEPTUAL}

En los últimos tiempos, ha tomado fuerza en la agenda de debate de la educación superior la necesidad de abordar la atención a las oportunidades de género en la organización de las instituciones educativas como universidades e institutos superiores, esto nos lleva la mirada hacia la concepción de las ideas de mujer, feminismo, género, etc., para ir definiendo las aristas del problema que se pretende indagar en esta investigación. Otro de los aspectos a tener en cuenta en la cuestión es la manifestación provocada o naturalizada de la discriminación de la que es objeto la mujer en la sociedad y en las relaciones académicas dentro mismo de las de las instituciones de educación superior.

Es relevante exponer los conceptos en los cuales se embarcan el análisis que se ha realizado; en cuanto al término género, se utiliza para hacer alusión a los rasgos o características psicológicas, sociales y/o culturales asociadas al sexo en determinados contextos ${ }^{3}$, lo que lleva a identificar

${ }^{3}$ Tobach, 2001, citado por Cardona y Martínez.

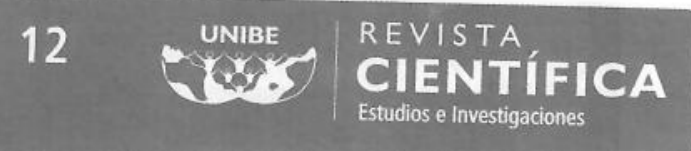


que pasan por procesos de construcción colectiva y a las cuales se va atribuyendo.

El tema de género fue instalado a partir del advenimiento del feminismo y ha presentado a través del tiempo las siguientes perspectivas ${ }^{4}$ :

- Feminismo liberal. Es la creencia de que las mujeres deberían tener los mismos derechos y oportunidades políticas, legales, económicas y educativas que los varones.(...)

- Feminismo cultural. Parte del supuesto de que las características de las mujeres y de los varones difieren y que, por lo tanto, habría que prestar una mayor atención y respeto a las cualidades especiales (...)

- Feminismo social. Sostiene que las desigualdades de género tienen su origen en las desigualdades económicas. (...)

- Feminismo radical. Parte de la idea de que las desigualdades de género derivan de la opresión del varón sobre la mujer. (...)

Cualquiera de las perspectivas de feminismo nos lleva a identificar unos postulados que remiten, por un lado, a diferencias concretas entre mujeres y hombres en la sociedad y por el otro lado, a luchas de igualdades construidas en el marco de la atención a las oportunidades de igualdad de género en los ámbitos tanto sociales como particulares de actuación de mujeres y hombres.

Siguiendo con la concepción de la idea de género, cabe resaltar que está relacionada con "los atributos, expectativas y normas sociales de conducta, y culturales asociados al hecho de ser mujer u hombre" . En atención a esta manera de concebir género, se pude asumir que se encuentra dentro del entramado de las relaciones sociales y las creencias que la compartan.

La idea de igualdad de género asumida en el trabajo se relaciona al "modo en que los atributos mencionados determinan la manera en que las mujeres y los hombres se relacionan entre sí y las consiguientes diferencias de poder entre unos y otros" . En esas maneras de relacionarse están puestas muchas expectativas con las que se confrontan mujeres y hombre al relacionarse en los diferentes ámbitos de sus vidas.

${ }^{4}$ Ens y Sinacore, 2001, citado por Carmona y Martínez

${ }^{5}$ Informe sobre el Desarrollo Mundial, 2012.

${ }^{6}$ Ibíd. 
Haciendo referencia a las discusiones planteadas en el país, se recurre a lo planteado por Clyde Soto (2011), con base en las investigaciones realizadas desde el Centro de Documentación y Estudios (CDE), "las mujeres $^{7}$ siguen siendo vistas como parte importante de la población paraguaya, incluso frecuentemente por sí mismas, como las serviha (las que están para servir), posiblemente como herencia de la falsa historia de amor de un mestizaje hecho como parte del proceso de dominación", estas expresiones presentan el contexto cultural e histórico en el cual se ha desarrollado la idea de ser mujer en la sociedad paraguaya, elementos de dominación y sumisión que marcan las expectativas de relacionamiento con el hombre y que se sigue en la actualidad solapada con el avance en el marco legal y el ensalzamiento de la valentía de la mujer paraguaya. Ante esta realidad se vuelve prioritaria iniciar el debate y el análisis sobre la igualdad de oportunidades en la propia organización de las instituciones de educación superior de tal manera a visibilizarlas e identificar como abordarlas para evitar una reproducción naturalizada en la sociedad.

\section{METODOLOGÍA}

Los objetivos de la investigación fueron las de diagnosticar la situación de género en la organización así como la de identificar el abordaje de las políticas de género en la universidad. Este trabajo es una ampliación del estudio anteriormente realizado, en el marco del Proyecto Diseño y Atención a las Oportunidades de Género en la Educación Superior sobre cultura organizacional con relación a la atención de oportunidades de género en la universidad llevada a cabo en el 2012 y los criterios e indicadores propuestos por la red para indagar sobre situación demográfica en la universidad y las políticas de atención a la temática de estudio.

El enfoque empleado es el mixto, lo cuantitativo para determinar la demografía en relación a género en la universidad y lo cualitativo para identificar la percepción sobre las políticas de atención a las oportunidades de género en la institución.

Las técnicas utilizadas fueron análisis documental para lo cual se ha diseñado el instrumento de guía del análisis para obtener datos relacionados a criterios e indicadores demográficos de la institución. La

${ }^{7}$ Se refiere a las mujeres paraguayas

\section{4

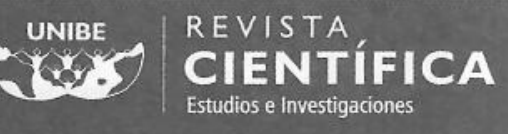


otra técnica aplicada fue la entrevista para la cual se recurrió a la guía de entrevistas a actores claves de la institución.

La población lo constituyeron tres miembros del Consejo de la Universidad, cinco coordinadores académicos de las facultades, diez profesores. El contexto en el cual se obtuvo los datos fue en las facultades ubicadas en la sede central de la universidad; los documentos que permitieron realizar el análisis documental fueron proveídos por el departamento de Recursos Humanos de la institución; los miembros de Consejo como los coordinadores y docentes dispusieron de sus tiempos en la universidad para acceder a las entrevistas previamente programadas.

Los datos fueron procesados en planillas Excel, una vez completado el registro de los datos, se procedió a obtener gráficos representativos de los resultados obtenidos. Con los datos obtenidos de las entrevistas fueron agrupadas las expresiones alrededor de los criterios e indicadores predefinidos para el estudio para luego realizar el análisis del discurso de las expresiones obtenidas de los y las entrevistadas.

\section{RESULTADOS}

\section{DESCRIPCIÓN DEMOGRÁFICA, POR GÉNERO}

Para realizar la descripción demográfica, se tuvieron en cuenta las cantidades de docentes, estudiantes, personal administrativo y la participación en los órganos de gobierno de la universidad.

Profesorado: PRESENCIA DE hOMBRES Y MUJERES, CATEGORÍA LABORAL

Profesorado de todas las carreras de la UNIBE, en sus dos locales.

Tabla 1. Cantidad de docentes de Grado y Posgrado

\begin{tabular}{|l|c|c|}
\hline \multicolumn{1}{|c}{ Categoría laboral } & \multicolumn{1}{c}{$\begin{array}{c}\text { Cantidad de } \\
\text { mujeres }\end{array}$} & $\begin{array}{c}\text { Cantidad de } \\
\text { hombres }\end{array}$ \\
\hline $\begin{array}{l}\text { Docentes de Grado y } \\
\text { Posgrado }\end{array}$ & 92 & 65 \\
\hline TOTAL & \multicolumn{2}{|c|}{157} \\
\hline
\end{tabular}


Los datos de cantidad de docentes por género son representados en la siguiente figura, en la cual se visibiliza con mayor claridad la preeminencia de mujeres en la organización.

Figura 1. Docentes de Grado y Posgrado.

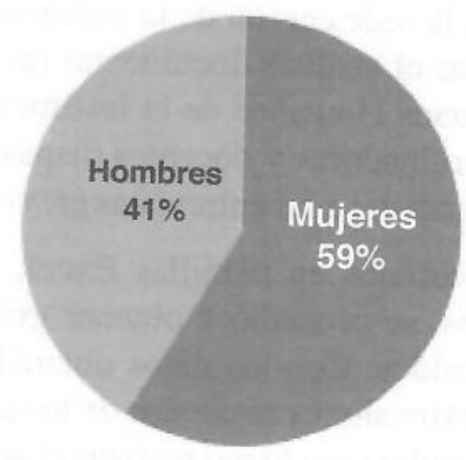

\section{Presencia de PRofesores y PROFESORAS EN LOS ÓRGanos DE GOBIERNo Y REPRESENTACIÓN}

Los órganos de gobierno en la institución los constituyen el Consejo de la universidad conformada por dos mujeres y dos hombres, en esta instancia de gobierno se identifica la paridad de género. El otro organismo de gobierno de la universidad es el conformado por las direcciones académicas de las facultades y del departamento administrativo; en relación de género distribuida de la siguiente manera:

Tabla 2. Cantidad de integrantes de Consejo Directivo

\begin{tabular}{|l|c|c|}
\hline \multicolumn{1}{|c}{ Categoría profesional } & Mujeres & Hombres \\
\hline Directivo & 2 & 2 \\
\hline Coordinación - Dirección Académica & 9 & 2 \\
\hline $\begin{array}{l}\text { Encargado/Jefe - Departamentos } \\
\text { Administrativos }\end{array}$ & 2 & 2 \\
\hline Total & \multicolumn{2}{c|}{19} \\
\hline
\end{tabular}

\section{6


Figura 2. Integrantes de Consejo Directivo

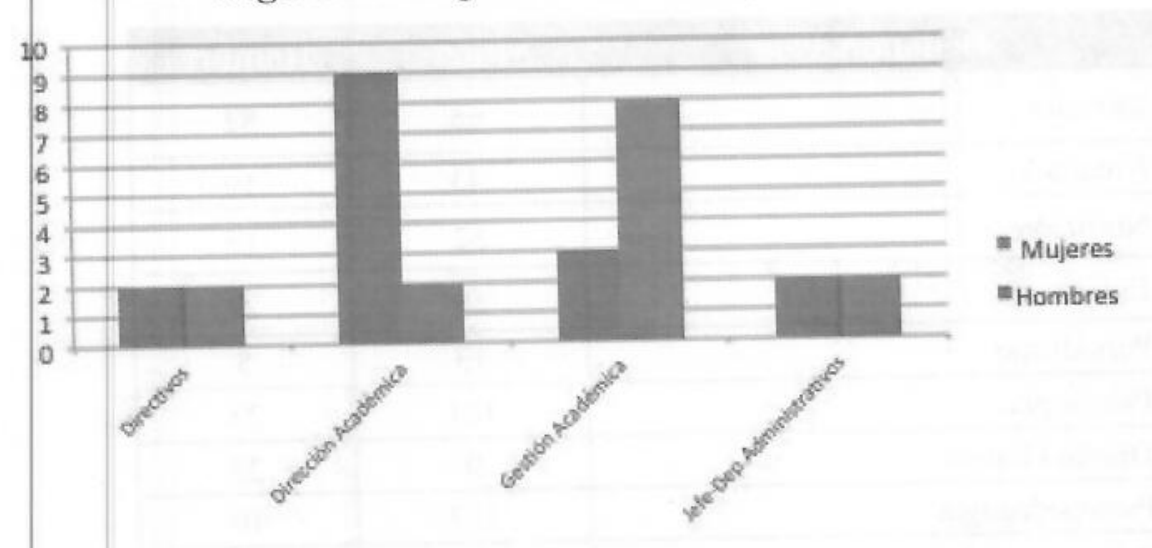

Estudiantes: presencia de hombres y mujeres

Los registros institucionales han permitido identificar la cantidad de estudiantes matriculados en estudios de Grado y Posgrado académico 2011 al 2012, en las modalidades presencial y a distancia (sistema b-learning). 
Tabla 3. Cantidad de matriculados por carreras de Grado

\begin{tabular}{|c|c|c|}
\hline Denominación & Mujeres & Hombres \\
\hline Derecho & 68 & 82 \\
\hline Notariado & 43 & 19 \\
\hline Nutrición & 52 & 13 \\
\hline Enfermería & 59 & 29 \\
\hline Periodismo & 13 & 5 \\
\hline Psicología & 101 & 23 \\
\hline Diseño Gráfico & 9 & 23 \\
\hline Psicopedagogía & 117 & 10 \\
\hline Psicomotricidad & 46 & 1 \\
\hline Comercio Internacional & 53 & 44 \\
\hline Marketing y Publicidad & 46 & 35 \\
\hline Ciencias de la Educación & 13 & 11 \\
\hline Licenciatura en Matemáticas & 8 & 6 \\
\hline Ingeniería en Informática & 1 & \\
\hline Administración de Empresas & 70 & 89 \\
\hline Contaduría y Auditoría Pública & 112 & 83 \\
\hline Análisis de Sistemas Informáticos & 14 & 64 \\
\hline Totales & 825 & 537 \\
\hline TOTAL & \multicolumn{2}{|c|}{1.362} \\
\hline \multicolumn{3}{|c|}{ Carreras a distancia (sistema b-learning) } \\
\hline Derecho & 2 & 9 \\
\hline Marketing y Publicidad & 2 & 0 \\
\hline Psicopedagogía & 17 & 3 \\
\hline Psicología Clínica & 8 & 2 \\
\hline Comercio Internacional & 1 & 0 \\
\hline Ciencias de la Educación & 3 & 3 \\
\hline Administración de empresas & 4 & 1 \\
\hline Contaduría y Auditoría Pública & 2 & 3 \\
\hline Totales & 39 & 21 \\
\hline TOTAL & \multicolumn{2}{|c|}{60} \\
\hline
\end{tabular}

\section{UNIBE


Figura 3. Matrículas por carreras de Grado

Lic. En Análisis de Sistemas

Contaduria y Auditoría Pública

Administración de Empresas

Ingenieria en Informática

Licenciatura en Matemáticas

Ciencias de la Educación

Marketing y Publicidad

Comercio Internacional

Psicomotricidad

Psicopedagogia

Diseh̊o Gráfico

Psicologia

Periodismo

Enfermeria

Nutrición

Notariado

Derecho

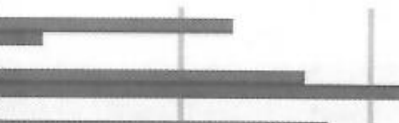

man

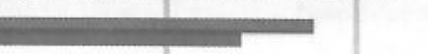

Hombres

Mujeres

En los resultados se puede evidenciar que de las 17 carreras analizadas se puede identificar que la mayoría cuenta con el predominio de las mujeres; solo en las carreras de Administración de Empresas, Análisis de Sistema Informáticos y Derecho se cuenta con mayoría de hombres. Cabe resaltar la diferencia significativa de la mayoría de mujeres en las carreras de Psicopedagogía, Psicología y Contaduría. En las demás carreras si bien prevalece la diferencia hacia las mujeres no resultan ser significativas.

A continuación se presentan los resultados obtenidos de la matrícula en los cursos de Posgrado 
Tabla 4. Cantidad de matriculados por cursos de Posgrado

\begin{tabular}{|l|c|c|}
\hline \multicolumn{1}{|c|}{ Denominación } & Mujeres & Hombres \\
\hline Doctorado en Educación & 30 & 18 \\
\hline Maestría en Educación & 13 & 37 \\
\hline Maestría en Salud Pública & 9 & 1 \\
\hline $\begin{array}{l}\text { Maestría en Neuropsicología Infantil y } \\
\text { Neuroeducación }\end{array}$ & 17 & 10 \\
\hline Maestría en Administración Hospitalaria & 30 & 14 \\
\hline Especialización en Didáctica Superior Universitaria & 24 & 3 \\
\hline $\begin{array}{l}\text { Especialización en Mediación y Resolución de } \\
\text { Conflictos }\end{array}$ & 44 & 25 \\
\hline Especialización en Administración Hospitalaria & 88 & 84 \\
\hline Diplomado en Mediación y Negociación & 5 & 1 \\
\hline Diplomado en Gestión Educativa & 79 & 48 \\
\hline $\begin{array}{l}\text { Diplomado en Administración Hospitalaria con } \\
\text { Enfasis en Atención Primaria a la Salud }\end{array}$ & 8 & 11 \\
\hline $\begin{array}{l}\text { Diplomado en Desafíos del Estado de Derecho del } \\
\text { Sigo XXI }\end{array}$ & 13 & 1 \\
\hline Diplomado en Gestión de Servicios Alimenticios & $\mathbf{3 8 0}$ & $\mathbf{2 5 9}$ \\
\hline Totales & $\mathbf{6 3 9}$ \\
\hline Total & & \\
\hline
\end{tabular}

\section{UNIBE $\mid \begin{array}{ll}\text { REVISTA } \\ \text { CIENTIFICA }\end{array}$


Figura 4. Matrículas por cursos de Posgrado

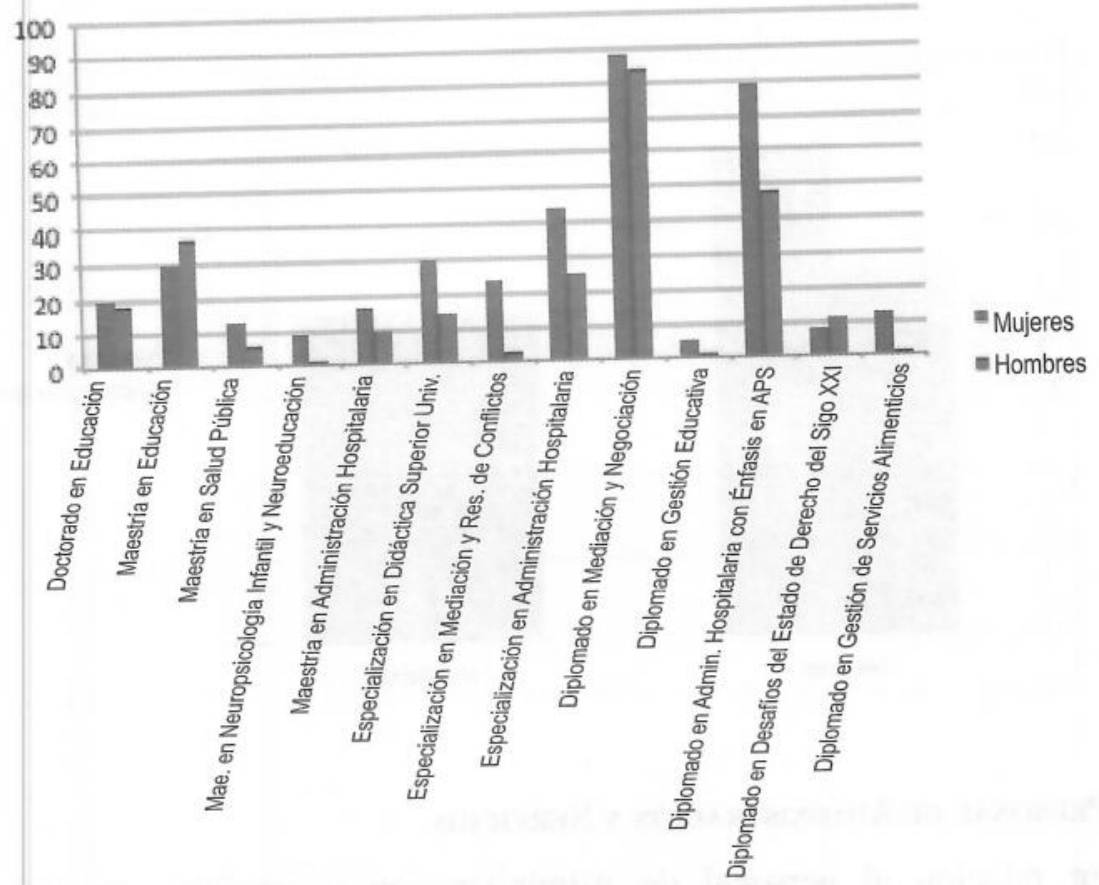

También se puede evidenciar que en los cursos de Posgrado se mantiene la prevalencia de la mayoría de mujeres. El único curso en el cual los hombres son la mayoría es en la Maestría en Educación y en la que tienden a equilibrar las cantidades con relación a las mujeres es en el Diplomado de Mediación y Negociación.

En relación a los premios otorgados a estudiantes de las diferentes carreras, en el periodo comprendido entre el 2011 al 2012, se puede evidenciar que fueron otorgados 8 a hombres y 9 a mujeres; en la categoría de sobresalientes fue otorgado a 8 hombres y 12 mujeres A continuación se presentan los datos en las figuras que graficas los resultados:

Tabla 5. Mejores egresados y egresadas. Estudiantes sobresalientes

\begin{tabular}{|l|c|c|}
\hline Denominación & \multicolumn{1}{l|}{ Mujeres } & \multicolumn{1}{c|}{ Hombres } \\
\hline Mejores & 9 & 8 \\
\hline Sobresalientes & 12 & 8 \\
\hline
\end{tabular}




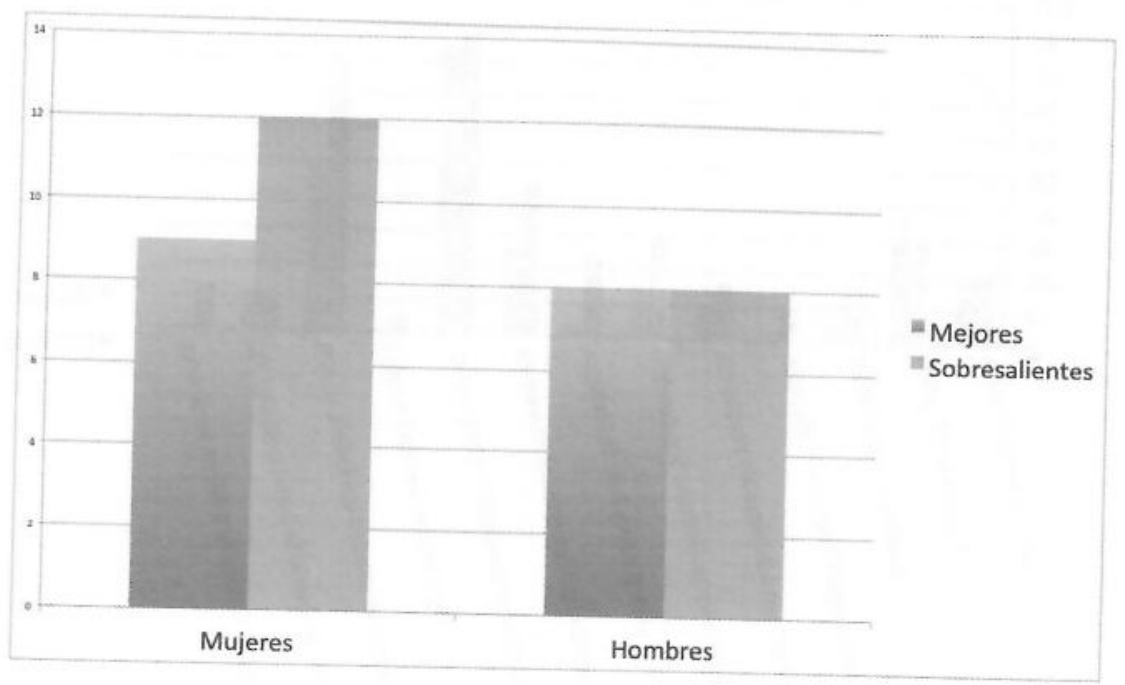

\section{Personal de Administración y Servicios}

En relación al personal de Administración y servicios, se siguen manifestando la prevalencia de las mujeres en relación a los hombres. A continuación se presentan los resultados.

Tabla 6. Personal de Administración y Servicios

\begin{tabular}{|l|c|c|}
\hline \multicolumn{1}{|c}{ Denominación } & Mujeres & Hombres \\
\hline Encargado/Jefe - Departamentos Administrativos & 13 & 5 \\
\hline Funcionarios Gestión Académica & 3 & 8 \\
\hline Funcionarios Gestión Administrativa & 11 & 7 \\
\hline Totales & $\mathbf{2 7}$ & $\mathbf{2 0}$ \\
\hline
\end{tabular}

\section{UNIBE REVISTA


Figura 6. Personal de Administración y Servicios

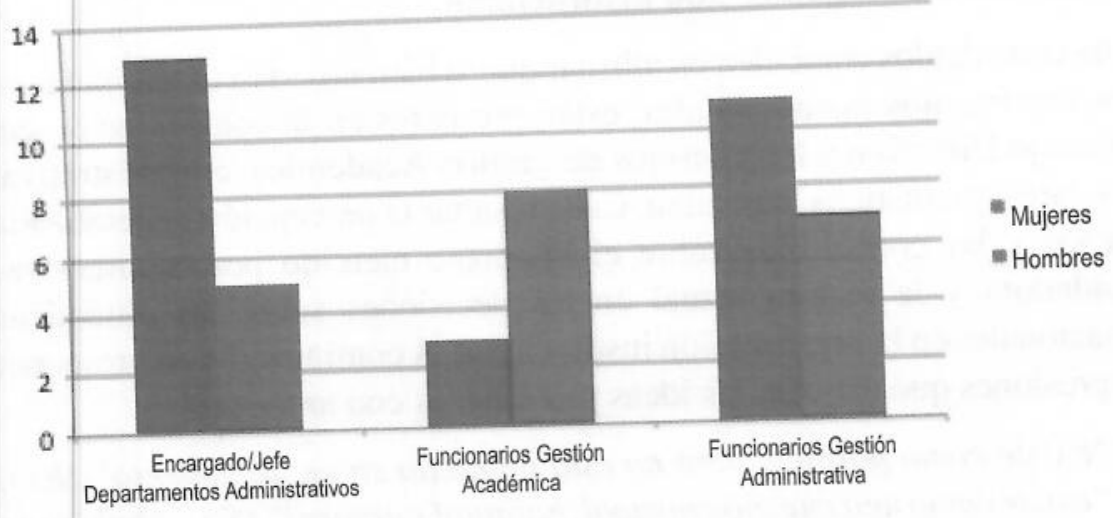

En todos los datos cuantificados se puede evidenciar que los y las integrantes de la comunidad educativa de la Universidad Iberoamérica están conformadas por la mayoría de mujeres, lo que podría estar influyendo significativamente en las decisiones que van tomando en la organización de la institución.

A continuación se presentan los resultados de las entrevistas aplicadas y con los resultados se podrían identificar mejor el compartimiento que va teniendo esta información otorgada por los resultados cuantitativos.

\section{Política universitaria SUB2}

La exploración realizada para obtener datos sobre las políticas que la universidad despliega para ser atendida las oportunidades de género se centraron en los criterios relacionados a las normativas, los mecanismos de regulación, mecanismos de conciliación familiar, el uso del lenguaje no sexista, sensibilización, asignaturas específicas de igualdad de género, materiales didácticos, participación de las mujeres en grupos de investigación, líneas de investigación en estudios de género y el fomento, promoción y difusión de tesis.

Los resultados se presentan a continuación organizados a partir de los criterios mencionados y los actores claves seleccionados para realizar las entrevistas. A continuación se presentan los resultados de los datos obtenidos de las y los miembros del Consejo Directivo de la universidad. 


\section{Normativas que garanticen la presencia equilibrada de mujeres y
hombres en la organización institucional.}

Los consultados coinciden en afirmar que si bien no están explicitadas en los documentos institucionales, están presentes en la conformación del Consejo Directivo y los consejos de gestión Académica, administrativa; los datos estadísticos lo revelan. Cabe resaltar la percepción generalizada en los y las consultadas sobre el liderazgo ejercido por la directorafundadora y la rectora actual en las decisiones sobre las dinámicas relacionales en la organización institucional. A continuación se presentan expresiones que denotan las ideas presentadas con anterioridad:

"Existe como política, pero no está implícita en un documento" (E1), "existe como una cuestión normal, habitual y natural" (E2), "habiendo sido fundada la Universidad por una mujer que ha sido fundadora de la Liga Paraguaya de los Derechos de la Mujer" (E3), "es un poco nuestra obligación de ir trabajando para las generaciones futuras, de tal manera que una reglamentación apoye a gestiones futuras que puedan permanecer este criterio o filosofía inicial de nuestra fundadora." (E1)

Se evidencia la falta de formalización de normativas que aseguren la presencia equilibrada de mujeres y hombres en la organización institucional, sobre todo desde una perspectiva de sostenibilidad del estilo de gobierno que hoy identifica la organización con los liderazgos legal y legítimamente identificados.

\section{Mecanismos de regulación de la representación equilibrada de mujeres y hombres en comisiones institucionales.}

Los resultados se relacionan con el anterior criterio, al no estar explicitadas en documentos institucionales y al contar con mayoría de mujeres, la representación equilibrada esta invisibilidad en la decisiones de conformación de los comités. De las expresiones recogidas en las entrevistas se pueden rescatar las siguientes manifestaciones que denotan la situación:

"Dado que se cuenta con recurso humanos mayoritariamente de mujeres, creo que este caso debería ser al revés debería incluir a los hombres para que haya realmente un equilibrio que es lo que se desea en una composición universitaria" (E1). "Solamente está el espíritu, el natural accionar de la institución se fomenta en la participación equitativa de los géneros, pero no existe por escrito" (E2). 


\section{Legislación de conciliación familiar}

Así como los anteriores aspectos indagados, la idea de conciliación familiar no se encuentra explícita en los documentos normativos de la institución. Para tomar decisiones relacionadas a permiso de maternidad se tiene en cuenta el Código Laboral. Ahora se ha iniciado el interés al tener en cuenta las siguientes expresiones:

Recién con la participación en este proyecto se ha incorporado la visión de visibilizar la familia como un aspecto esencial del abordaje de género. (E1) "estamos haciendo fuerza para que eso se instale oficialmente" (E2).

También surge la necesidad de interiorizarse hacia el abordaje de este aspecto de la igualdad de oportunidades de género en la organización, según se denota en las siguientes expresiones:

"Me interesaría informarme estar en conocimiento para saber cómo incluirlo en la Universidad". "Constituye uno de los desafios a ser afrontados desde análisis de la propuesta teórica a la toma decisiones para instalar mecanismo a ser implementado". (E1)

En relación a la concreción de campañas específicas dirigidas a toda la comunidad universitaria con el objetivo de promover la cultura de la corresponsabilidad en el ámbito de la vida familiar, una de las consejeras explica de manera detallada los esfuerzos y las intenciones que cimentan la necesidad de fortalecer los vincular universidad-familia:

Se está trabajando, pero sin una política preestablecida. No es un ejercicio tan común.

Pero sí en el diálogo, en las entrevistas, que es lo que tienen mayormente los estudiantes, casi un coaching ontológico, que hace la universidad con los alumnos.

Se les va reconociendo su responsabilidad personal e integral en una familia, que son un equipo y que naturalmente requieren apoyarse mutuamente.

Se incentiva en los periodos de pre ingreso cuando se hace la orientación a la carrera, a la vida universitaria y cuando entran en el periodo de tesis, porque son procesos en los cuales la persona requiere de un tiempo personal dedicado al estudio, en su propia casa y muchas veces eso no se comprende (...), para lo cual necesita plantear este tiempo de corresponsabilidad con su familia, de funciones en su 
familia para que ella pueda tener tiempo, no libre, sino dedicado al estudio en su hogar.

Estos son planteamientos que normalmente yo los hago en forma personal con los estudiantes y creo que hay que institucionalizarlos. Esto es muy importante, trabajar desde el vamos, cuando hablan de la familia, siempre se pone como parte integral, en toda la proyección que tienen los alumnos, desde su ingreso a partir de la orientación, del juramento, con la presencia de su familia, entender que este nuevo proyecto de vida, constituida dentro de una familia, será posible si es que existe una colaboración, no significa que se va a desligar de sus responsabilidades en la familia, sino que de algunas tareas tendrá que abstenerse y otras tendrá que compartir con otras personas, hablando desde personas solteras que trabajan y estudian o personas con familias, llámese dentro de un matrimonio o que tengan hijos.

Esa mirada yo me encargo de darles, por mi propia experiencia de vida, yo sé que si no se organizan bien no van a llegar a la meta de estudio, pero también pueden no llegar a su realización personal, en la familia. Los dos extremos no son buenos, ni que se dedique excesivamente al estudio ni que abandone todo porque se está llenado de responsabilidades familiares. Debe haber un equilibrio en todo.

Eso tenemos que trabajar a quienes tenemos en la casa, que son nuestros estudiantes; yo noto que eso cala muy profundamente en la vida personal de los alumnos y les son muy útiles estas orientaciones en el proceso de su desarrollo.

También cuando se dan casos de deserciones. Las deserciones se deben justamente a situaciones en las cuales no se cuenta con ese apoyo familiar, entonces ahi se hace como una terapia con cada uno de los alumnos, escuchándolos por sobre todas las cosas, y tratando de juntos ir encontrando la salida a la realidad que a él o a ella le corresponde enfrentar. Porque no se puede hacer una fórmula para todas las cosas, entonces, en ese sentido, señalo que en ese proceso es muy importante Bienestar estudiantil y las Coordinaciones de carreras también.

Las manifestaciones expuestas denotan un alto interés por vincular los aspectos familiares con las demandas de la formación universitaria, el gran esfuerzo que queda por realizar es concretar dichas acciones en procedimientos institucionales, en programas a ser desplegados en cada

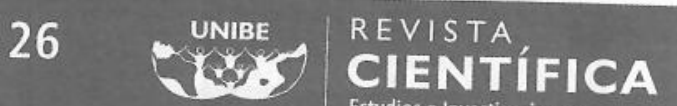 Estudios e Investigaciones}


una de las facultades de la universidad para que lo vuelvan sostenibles en el tiempo y se pueda ir incorporando mejoras a partir de la implementación de acciones específicas.

Lineamientos, normativas o disposiciones que eviten el uso del lenguaje no sexista en las comunicaciones internas y externas de la universidad

El uso del lenguaje no sexista no se encuentra explícito en las normativas nacionales; en la universidad, así como los anteriores aspectos, no se tiene formalizado en los documentos institucionales, según los y las ingresantes del consejo de la universidad. Se encuentra en proceso de ser incorporado en las normativas. Las siguientes manifestaciones ilustran las ideas de los y las consultadas:

"Existe legislación en Paraguay, y como tal nos sometemos a ella, pero es muy poco conocida esa legislación, requiere mayor difusión y mayor conocimiento por parte de todos".(E1) "No sentimos esta necesidad, siendo una universidad constituida por la mayoría. No se siente esa necesidad; probablemente eso no quiere decir que no tengamos que ir haciendo porque el proyecto de universidad como tal puede darse con otras personas también y es importante dejar el cimiento, para que eso sea respetado y no muera esa filosofía institucional y no se pierda en el camino, en el tiempo de vida de una institución. (E1)

\section{Campañas de sensibilización}

Las campañas que se realizan en la universidad, dirigidas a estudiantes y docentes, orientadas a combatir estereotipos de género y a promover la igualdad de oportunidades, están vinculadas con las alianzas estratégicas que la universidad tiene con la Secretaría de la Mujer, organismo dependiente de la Presidencia de la República y a la fundación Kuña Aty. Ahora la universidad cuenta, con:

"La campaña más fuerte es con la Beca Doña Nidia, que se otorga a las mujeres cabezas de HOGAR”. (E1)

"En el tema mujer se trabajó en una campaña con niños en situación de calle, pero se lo hizo específicamente con las mamás cabezas de hogar y se les dio un proyecto de capacitación, de emprendimiento; se le becó para terminar el colegio. Muy interesante". (E2) "Se vive una fuerte relación y un lugar preponderante de la mujer”. (E3) 
Cabe resaltar que en la organización se percibe una fuerte sensibilidad hacia la presencia de la mujer, pero la idea de igualdad de oportunidades dentro de ella se diluye en la dinámica cotidiana. Los esfuerzos están más vinculados hacia la promoción de sensibilización dirigida a la sociedad en general.

Los siguientes resultados fueron obtenidos de las entrevistas aplicadas a docentes y coordinadores de carreras de la universidad. Se ha seleccionado a docentes que cuentan con la mayor permanencia de carga horaria en la institución. Estos son resultados:

\section{Asignaturas específicas de igualdad de género}

Las entrevistas reportan como resultado que los y las docentes manifiestan, en su mayoría, que no cuentan con información sobre la existencia de asignaturas específicas referentes a la igualdad de género en las carreras de Grado, pero se aborda el tema de igualdad de género dentro de los proyectos o programas de las asignaturas.

Según lo manifestado por las coordinaciones de las diferentes carreras, se ha identificado que se cuentan con asignaturas vinculadas al eje temático de género. Esto en las carreras de Psicología y en la de Derecho; y en el Posgrado, en la especialización en Mediación y Resolución de Conflictos. Además, se ha iniciado una revisión de los currículum en el marco de los procesos de autoevaluación de las carreras, situación que consideran oportuna para incorporar como asignaturas o como eje temático de módulos afines al tema.

En proyecciones se encuentra la concreción de un curso de especialización sobre el tema de la situación de género en las instituciones y en especial, en las instituciones educativas; el proyecto está relacionado con un programa del Ministerio de la Mujer.

\section{Elaboran materiales}

En relación con la producción de materiales didácticos, los y las entrevistadas manifestaron, en su mayoría, que estaban sujetos a las decisiones autónomas de docentes.

\section{Participación de las mujeres en grupos de investigación}

En relación a la participación de las mujeres en grupos de investigación, cabe resaltar que en los últimos años se fue fortaleciendo la conformación de los equipos de investigación a partir de proyectos específicos.

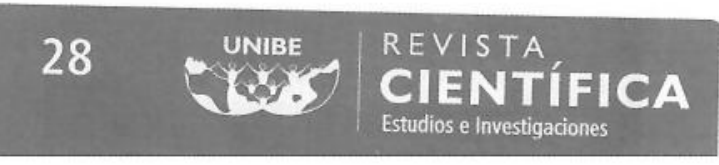


Actualmente son los grupos de investigación los que cuentan con paridad de mujeres y hombres, pero no con intencionalidad, sino como una de la situación coyuntural del interés de investigación.

\section{Convocatorias de subvención pública}

Existen convocatorias de subvención pública para proyectos I+D (Investigación y Desarrollo) que incluyan la perspectiva de género, lo realiza el CONACYT. Aún no son suficientes como para asumir como cantidad importante que dé cuenta del estado del abordaje de género en el país. Cabe resaltar que no se considera esencial la participación de las mujeres en grupos de investigación como criterio para la concesión de ayudas a proyectos.

\section{Líneas de investigación en estudios de género}

La línea de investigación que está en proceso de incubación es la relacionada con el abordaje de género en la legislación del país, en la carrera de Derecho, y en las demás no se cuenta con información. Una de las consejeras ha manifestado:

"En este momento estamos iniciando el proceso para presentarnos a una convocatoria del CONACYT en relación con una investigación sobre momentos de ocio de la mujer".

Otra estrategia que se instala es la inclusión de la temática de género, como una de las líneas a ser desarrolladas en las disertaciones en los Foros de Investigaciones de Salud y Educación, que se realizan en el mes de noviembre cada año.

Así mismo, se ha tomado la decisión de destinar un apartado de la Revista Científica a la publicación de artículos sobre la temática de igualdad de oportunidades de género. Ya sean productos de estudios monográficos o estudios realizados por los docentes.

\section{Fomento, promoción y difusión de tesis}

Según los consultados, no se cuentan con informaciones precisas sobre el desarrollo de tesis en esta temática. 


\section{DISCUSIÓN}

La presentación de los resultados permite identificar aspectos relevantes que ameritan su discusión y análisis, como los expuestos a continuación:

La prevalencia de la invisibilización de la atención a la igualdad de oportunidades de género en la universidad, este aspecto relevante en la discusión sobre las relaciones entre las personas, en la universidad no se considera un problema. La percepción es que no se identifican tratos desiguales entre mujeres y hombres en toda la estructura organizativa de la universidad. Una de las razones que podrían estar incidiendo en la manera de interpretación de la situación de género en la organización, según se comprobó en este estudio, es que la mayoría de las personas que interactúan en la institución son mujeres.

Se pudo comprobar la falta de formalización, de explicitación en documentos institucionales el abordaje de igualdad de oportunidades de género; este hecho puede estar en relación directa con lo mencionado anteriormente sobre la mayoría de mujeres en la organización. En las manifestaciones obtenidas en las entrevistas, se ha identificado la ausencia de tensiones que provengan de disputas explícitas sobre la igualdad de oportunidades dentro de la organización de la universidad.

Si bien es clara la posible influencia de la mayoría de mujeres en la organización, lo que queda por indagar en profundidad es ¿cuál es la representación que las mujeres de la universidad tienen sobre el abordaje de género y su relación con la atención a las oportunidades en la educación superior?, sobre todo porque de manera implícita se podría estar accionando en parámetros, principios y creencias contrarias a la igualdad de oportunidad de género.

Lo que se revela en los datos cuantitativos es que en las carreras de Derecho, Análisis de Sistemas, Administración y la maestría en Educación, son la mayoría hombres. Las primeras son profesiones desempeñadas culturalmente por hombres en el ámbito laboral y no hay indicios de que esta diferencia tenga vinculación con desigualdades en la condición de estudiantes. En cuando a la maestría, es llamativa la presencia mayoritaria de hombres, es un ámbito interesante para indagar las razones de este fenómeno.

Uno de los aspectos que se percibió como llamativo por los y las entrevistadas ha sido la incorporación en la agenda de atención a las oportunidades de género el tratamiento de la conciliación de la familia

\section{UNIBE REVISTA $_{\substack{\text { CIENTÍFICA } \\ \text { Estudios envestigaciones }}}$}


con el ámbito laboral; esto se presenta como un ámbito que requiere su profundización teórica y el diseño de estrategias para su incorporación en las normativas institucionales. Esto significaría además incorporar en el debate de la educación superior este aspecto que afecta a las mujeres que trabajan en las universidades, institutos superiores y a las del tercer nivel del sistema educativo nacional.

\section{CONCLUSIÓN}

El estudio realizado ha permitido identificar la necesidad de incorporación en los documentos institucionales el abordaje de la atención a las oportunidades de igualdad de género, para lo cual se requerirá de instalar un proceso participativo de estudio, debate y toma de decisiones sobre el tema a ser incorporado en las normativas institucionales, así como la definición de políticas y estrategias de conciliación de la universidad, ámbito laboral con la familia.

Se requiere profundizar en el fortalecimiento de las líneas de investigación sobre la temática en género para proveer a la sociedad académica de conocimiento científico que permita constituirse en base para la toma de decisiones que pudieran impulsar la atención a las oportunidades de género en la educación superior del país.

Ante el desafío, es llegar a constituirnos en una institución de educación superior que cuente con una organización, líneas de investigación y difusión de la atención a las oportunidades de género modelo dentro de la educación superior en el país, se requerirá definir una planificación estratégica para instalar, tanto en los documentos institucionales como en la propia cultura de la universidad.

\section{BIBLIOGRAFÍA}

Banco Mundial. (2012). Informe sobre el desarrollo mundial. Igualdad de género y desarrollo. Panorama general. Banco Internacional de Reconstrucción y Fomento/Banco Mundial. Washington. wdronline. worldbank.org/worldbank/a/langtrans/65. Consultado el 29.12.2012

CAPrile, M. (Coord.) (2012). Guía práctica para la inclusión de la perspectiva de género en los contenidos de investigación España: CIREM 
FERnÁNDEZ, P. (2007). Igualdad de oportunidades y conciliación. Una visión multidisciplinar Jaén: Universidad de Jaén.

GuZmán, Virginia y Corbalán, Graziella. (2003). La institucionalidad de género en un contexto de cambio de gobierno: el caso Paragua CEPAL. Proyecto CEPAL/DAW/ Naciones Unidas, Cuenta para el Desarrollo "Gobernabilidad Democrática e Igualdad de Género en América Latina y el Caribe". Págs. del 5 al 55. Sitio web: http://books.google.com.py/ books? $\mathrm{id}=\mathrm{dLvj} 2 \mathrm{yxb}$ ArYC \&pg=PA55\&dq=line+bareiro+paraguay \&h

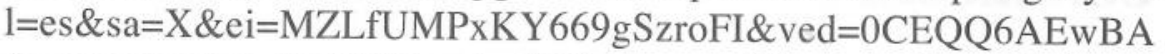
$\# \mathrm{v}=$ onepage $\& \mathrm{q}=$ line $\% 20$ bareiro\%20paraguay $\& \mathrm{f}=$ true. Consultado el 29.12.2012.

Martínez Ruzz, María Ángeles; y Merma Molina, Gladys (2012). Redes de Formación e Innovación en Igualdad de Oportunidades. Una aproximación al diagnóstico estratégico de la igualdad de oportunidades de género en la educación superior en Hispanoamérica. Conferencia presentada en el Primer Congreso de investigadores de la Universidad Iberoamericana.

Rebollo, M. A. (coord.) (2006). Género e interculturalidad: educar para la igualdad Madrid: Editorial La Muralla, S.A.

Rodríguez, C. (2006). Género y currículo. Aportaciones del género al estudio de práctica del currículo. Madrid: Akal.

Soто, Clyde (2009). Marcas culturales para las mujeres en la sociedad paraguaya. Ponencia presentada en: Primer Foro Internacional del Bicentenario. Comisión del Bicentenario Asunción, 6 y 7 de agosto de 2009. http://web.ua.es/es/proyectogenero/proyecto-genero-de-launiversidad-de-alicante.html

Torres, I. (coord.) (2005). Miradas desde la perspectiva de género. Estudios de las mujeres. Madrid: Narcea.

\section{UNIBE $\mid \begin{aligned} & \text { REVISTA } \\ & \text { CIENTÍFICA }\end{aligned}$}

